\title{
Effects of working memory load on visual selective attention: behavioral and electrophysiological evidence
}

\author{
Nikki Pratt ${ }^{1}{ }^{*}$, Adrian Willoughby ${ }^{1}$ and Diane Swick ${ }^{1,2}$ \\ General Medical Research, VA Northern California Health Care System, Martinez, CA, USA \\ 2 Department of Neurology, University of California Davis, Davis, CA, USA
}

Edited by:

Leon Y. Deouell, The Hebrew University of Jerusalem, Israel

Reviewed by:

Chien-Te Wu, National Taiwan

University College of Medicine, Taiwan

Sander Martens, University of

Groningen, Netherlands

Yigal Agam, Harvard Medical School,

USA

\section{*Correspondence:}

Nikki Pratt, VA Northern California

Health Care System, 150 Muir Road,

151-I, Martinez, CA 94553, USA

e-mail: nikki.pratt@va.gov
Working memory and attention interact in a way that enables us to focus on relevant items and maintain current goals. The influence of working memory on attention has been noted in several studies using dual task designs. Multitasking increases the demands on working memory and reduces the amount of resources available for cognitive control functions such as resolving stimulus conflict. However, few studies have investigated the temporal activation of the cortex while multitasking. The present study addresses the extent to which working memory load influences early (P1) and late (P300) attention-sensitive event-related potential components using a dual task paradigm. Participants performed an arrow flanker task alone (single task condition) or concurrently with a Sternberg memory task (dual task condition). In the flanker task, participants responded to the direction of a central arrow surrounded by congruent or incongruent arrows. In the dual task condition, participants were presented with a Sternberg task that consisted of either four or seven consonants to remember prior to a short block of flanker trials. Participants were slower and less accurate on incongruent versus congruent trials. Furthermore, accuracy on incongruent trials was reduced in both dual task conditions. Likewise, P300 amplitude to incongruent flanker stimuli decreased when working memory load increased. These findings suggest that interference from incongruent flankers was more difficult to suppress when working memory was taxed. In addition, P1 amplitude was diminished on all flanker trials in the dual task condition. This result indicates that top-down attentional control over early visual processing is diminished by increasing demands on working memory. Both the behavioral and electrophysiological results suggest that working memory is critical in maintaining attentional focus and resolving conflict.

Keywords: dual task, working memory, ERPs, attention, P1, P300

\section{INTRODUCTION}

Imagine driving along a busy suburban street with the radio blaring. While stopped at a light, the DJ on the radio announces that the 100th caller will win free tickets to a show you have wanted to see and then gives the phone number to call. As the light turns green, you rehearse the number in your head and drive through a complicated intersection with confusing signs. You take a wrong turn and end up on the freeway instead of the parking lot, where you had intended to stop and call the radio show number. Because you were focused on rehearsing the phone number and not attending to street signs, you were unable to call the radio for the tickets.

This scenario illustrates the difficulties of multitasking in everyday life. More specifically, attention was diminished when working memory capacity was loaded. Attention is regulated by a dynamic network that responds to both external events and internal goals (Yantis, 2000). Attention may be focused on specific visual features and objects driven by salient external events in an automatic fashion (bottom-up), or by internal expectations requiring cognitive control (top-down). Top-down attention influences the selection of visual stimuli based on previous experience and current goals, while filtering out distractor stimuli (Hopf and Mangun, 2000; Corbetta and Shulman, 2002; Bledowski et al., 2004; Lavie et al., 2004). Working memory plays a critical role in guiding these top-down attentional processes by keeping current goals in mind (Downing, 2000; de Fockert et al.,
2001; Soto et al., 2005). But what happens when working memory is filled with items that are irrelevant for the goals required by a secondary task? In the example above, working memory was loaded with a seven-digit phone number and diminished the driver's capacity to attend to road-signs. Performance in this dual task scenario requires the coordination of multiple cognitive processes, including working memory, selective attention, and conflict resolution.

Lavie and colleagues (de Fockert and Lavie, 2001; Lavie et al., 2004; Lavie, 2005; Lavie and de Fockert, 2005) have demonstrated that increasing the demands on working memory reduces the ability to ignore irrelevant stimuli. They used a dual task design to manipulate the amount of information stored in working memory (one or six digits) while participants performed a flanker interference task (Lavie et al., 2004). In the letter flanker task, reaction times (RT) increased significantly when the flanker was incompatible with the target. This flanker interference effect was greater when working memory load was high relative to when it was low (de Fockert et al., 2001; Lavie et al., 2004; Lavie, 2005). This result shows that working memory is essential for overcoming response conflict and for optimal selective attention performance.

Few studies, however, have examined the effects of working memory load on neural activity in attention-sensitive visual regions during dual task performance. In one functional magnetic resonance imaging (fMRI) study, participants were presented with 
a memory set followed by names of famous individuals superimposed over either compatible or incompatible faces (de Fockert et al., 2001). Activity in prefrontal cortex (PFC) was greater in the high working memory load condition than the low load condition. Furthermore, activity in the fusiform gyrus and extrastriate visual cortex was greater when distractor faces were present in the high load condition, relative to the low load condition. Thus, a high working memory load increased neural processing of distractors and resulted in greater behavioral interference.

Although $\mathrm{fMRI}$ identifies specific regions in the brain that are active in cognitive processing, it provides poor temporal resolution. Therefore, it is unclear whether activation reflects early or late changes in attention. The timing of cortical responses is a critical aspect of cognitive control and attention systems. Event-related potentials (ERPs) provide precise information regarding the timing of neural activity (Hillyard and Anllo-Vento, 1998). The present study adapted the design from Lavie et al. (2004) and examined the time-course of neuronal processing using ERPs during an attention task embedded within the delay period of a standard item recognition task. The number of items to be remembered was manipulated, and a single task condition without the memory load was also included. The neuronal time-course of changes in attention following working memory load was examined by investigating two specific ERP components: the P1 and the P300.

The $\mathrm{P} 1$ is an early visual component which peaks at approximately $80-120$ ms over occipital regions. Dipole modeling and combined $\mathrm{fMRI}$ /ERP studies have suggested that the P1 is generated in extrastriate cortex (Di Russo et al., 2001). P1 is larger to stimuli that appear at an attended location compared to stimuli that appear at an unattended location (Hillyard and Anllo-Vento, 1998; Hillyard et al., 1998). In addition, researchers have noted that the P1 amplitude is smaller when perceptual load demands increase. For instance, increasing the number of irrelevant stimuli within a display caused a diminished $\mathrm{P} 1$ over the parietal-occipital region (Handy et al., 2001). Handy et al. (2001) concluded that an increase in perceptual load reduces the capacity to attend to specific attributes within the visual field. In addition, other researchers found that previously encoded spatial locations increase the attentionbased component P1 (Awh et al., 2000). The authors concluded that spatial attention changes early visual processing by sustaining activation of locations in working memory (Awh et al., 2000). In contrast to these experiments on increasing perceptual load and the underlying effects on early selective attention, little is known about the effects of cognitive load on P1 amplitude.

Finding a decrease in P1 amplitude under conditions of cognitive load, such as working memory maintenance, would suggest that less attention was allocated for target detection in the secondary task. However, most studies have not employed dual task paradigms. The focus of prior research has been on the encoding, maintenance, or retrieval phases of working memory tasks. Task difficulty is manipulated by increasing the number or category of items that must be remembered. These studies have suggested that the P1 (and other early components) can be influenced by attention-driven, top-down modulation of visual processing (Gazzaley et al., 2008). However, to the best of our knowledge, no studies have investigated the effects of working memory load on the P1 elicited during a secondary, unrelated executive control task.
Another common ERP component related to attention is the P300, a positive wave from approximately $300-650 \mathrm{~ms}$ that is maximal over the central-parietal region. The P300 is associated with shifts in attention that update representations in working memory (Polich and Kok, 1995).P300 amplitude decreases when attention is directed away from the current target (Duncan et al., 2009). The P300 is also sensitive to demands placed on working memory (Wintink et al., 2001). Wintink et al. (2001) found that the P300 decreased by one microvolt over the parietal region for each additional item placed in working memory in an n-back task. In another n-back task, researchers found that the P300 also decreased when more items were maintained in working memory (Watter et al., 2001). These authors argued that the n-back task is a type of dual task paradigm requiring participants to update working memory as well as match current stimuli to encoded items in working memory. Therefore, the P300 may be an index of attention processing and working memory demands.

The goal of the current experiment is to examine the importance of working memory and cognitive control processes for the resolution of response conflict in a demanding visual attention task. Just like the scenario in the beginning, it did not matter whether the phone number rehearsed was accurately remembered. Rehearsing the phone number diminished visual attention to nearby roadsigns, and the caller was never able to enter the contest. To investigate the extent to which working memory load decreases attention, we extended previous research (Lavie et al., 2004) by employing a verbal working memory task and examining the subsequent effects on conflict resolution in a visual selective attention task. Specifically, we investigated the effects of cognitive load on selective attention using concurrent Sternberg memory and flanker interference tasks. Both behavioral and ERP responses were used to detect changes in attentional control due to varying demands on working memory. We used a modified version of the Eriksen flanker task (Eriksen and Eriksen, 1974) to examine selective attention and cognitive control processes. Irrelevant flanker stimuli could be either congruent or incongruent with the central target. Participants performed the flanker task alone and in conjunction with a working memory task. Similar to the studies reported by Lavie et al. (2004, 2005), we varied cognitive load by using a memory set containing either four or seven items. These items then had to be maintained over the delay period during which a flanker task was performed. Based on previous behavioral findings, it was expected that incongruent flankers would increase RTs, specifically in the high working memory load condition (Lavie et al., 2004, 2005).

We also predicted that high working memory load would affect attentional ERP responses over the occipital and parietal regions. Similar to Wintink et al. (2001), we expected to find a decrease in P300 amplitude over the parietal region when cognitive load was increased. In addition, we expected that working memory demands would tax PFC regions that send top-down cortical projections to the visual cortex, thereby influencing early attentional processing (de Fockert et al., 2001; Krawczyk et al., 2007). Therefore, we expected to find significant changes in $\mathrm{P} 1$ amplitude, indicating that early attentional processing in the visual cortex is diminished in the high working memory load condition. Specifically, if items are being maintained in working memory, then fewer neural resources in PFC will be available for the flanker task, thereby resulting in decreased ability to resolve response conflict (Lavie and De Fockert, 
2005). No study has investigated such effects on both the P1 and P300 attention components using a dual task design that manipulates cognitive load, so the current results will provide novel insights into the temporal parameters of top-down attentional control.

\section{MATERIALS AND METHODS PARTICIPANTS}

Sixteen healthy, young participants (aged 18-30, 8 female) completed the dual task experiment, none of whom reported a history of neurological or psychological disorder, or significant substance abuse. Due to excessive noise, 1 subject was excluded from the analysis; the data from the remaining 15 are reported here. The experimental protocol was approved by the Institutional Review Board of VA Northern California Health Care System, and all participants gave informed consent prior to beginning the experiment. They were paid for transportation expenses plus $\$ 20 / \mathrm{h}$ for their participation.

\section{PROCEDURE}

Participants were tested in a darkened, sound-attenuated room. EEG was recorded from 48 electrode sites positioned according to the 10/20 system (Jasper, 1958). All participants were instructed to fixate at the center of the screen and to blink as little as possible. Each participant completed both single and dual task flanker conditions as well as a single task Sternberg condition. The order was counterbalanced across participants. In both the single and dual task conditions, flanker stimuli were presented for $200 \mathrm{~ms}$. Inter-trial interval varied randomly between 600 and $800 \mathrm{~ms}$ after the participant's response. If no response was made, the trial terminated after $900 \mathrm{~ms}$. All tasks were divided into 10 blocks of trials, each block lasting about $3 \mathrm{~min}$. The total test time required was approximately $2 \mathrm{~h}$.

\section{STIMULI AND TASKS}

\section{Single task condition, flanker}

Participants responded with a button press to indicate whether the central arrowhead pointed to the left or the right. Flanking arrows, positioned either above, below, or both above and below the central arrow, could point in either the same (congruent) or different (incongruent) direction (see Figure 1). Forty percent of trials had congruent flankers; $60 \%$ of trials had incongruent flankers. The asymmetrical and symmetrical flankers were equally presented in the congruent and incongruent conditions. Participants were instructed to respond as quickly and accurately as possible to the central arrow. Each participant completed 10 blocks of 60 flanker trials.

\section{Sternberg condition}

Participants were instructed to remember either four or seven consonants. In the set size 4 condition, the stimuli were presented for $2000 \mathrm{~ms}$, whereas the set size 7 condition was presented for 3500 (i.e., a 500-ms encoding time for each letter in the memory set). After an 8.5-s delay, another consonant was presented. Participants responded with a button press to indicate whether or not this item was from the memory set. Half of the trials had probes that were from the memory set and half were not. Each block contained 10 Sternberg trials randomly selected to present half the trials with four items and the other half with seven items. There were a total of 10 blocks.

\section{Dual task condition}

In the dual task condition, participants were required to perform the Sternberg memory task in addition to the flanker task (whose parameters remained the same). Participants were presented with a set of either four or seven consonants to be remembered over a delay period as described above for the Sternberg condition. Between 300-500 ms following presentation of the memory set, the flanker trials began. Nine flanker trials were presented. At $500 \mathrm{~ms}$ following the final flanker trial of the block, participants were presented with a probe item (a consonant) and responded with a button press to indicate whether this item was in the previous memory set. On half the trials the probe was a member of the memory set, on the other half, it was not. The probe trial terminated once a response was made. Each participant completed a total of 90 flanker trials in each of the 10 blocks (total: 900 flankers). The Sternberg task was randomly selected to have five sets of four items and five sets of seven items for each of the 10 blocks (total: 50 sets of $4 ; 50$ sets of 7).

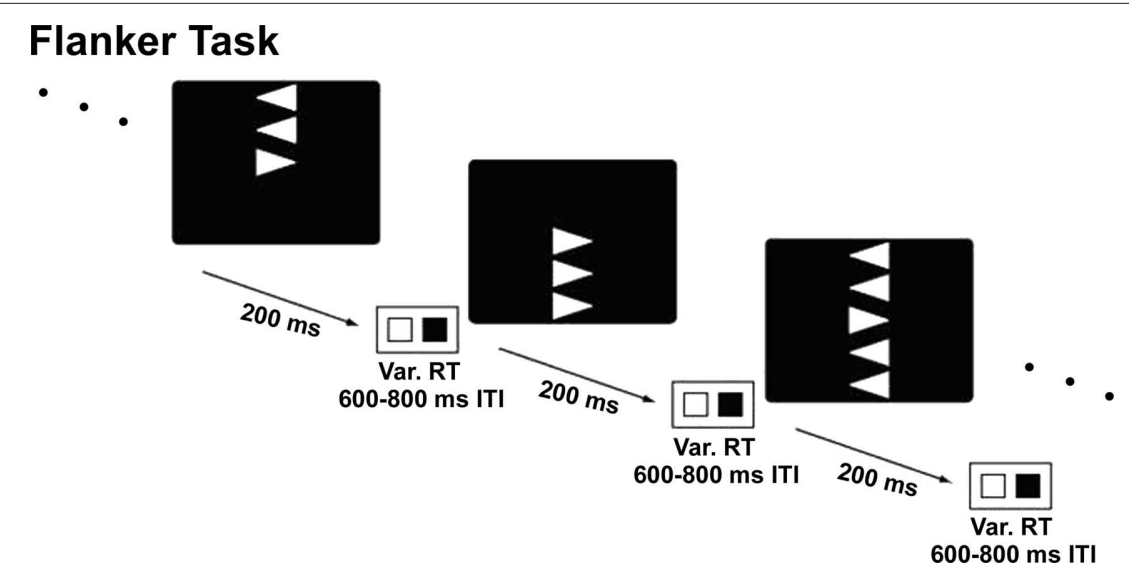

FIGURE 1 | Flanker task design. Participants responded to direction of the central arrow using a two-button response. Flanker arrows could be either congruent or incongruent and above, below, or both above and below the central arrow. 


\section{EEG RECORDING}

The EEG was recorded from participants using an SA Instrumentation amplifier and DataPAC 2000 software. The EEG was sampled at $256 \mathrm{~Hz}$ using an online low-pass filter of $100 \mathrm{~Hz}$ and a high pass of $0.1 \mathrm{~Hz}$. Impedances were maintained below $10 \mathrm{k} \Omega$. Electrodes were initially referenced online to the left mastoid. Eye artifacts (e.g., blinks, movements) were monitored with four EOG electrodes and corrected offline. Offline analysis was completed with EEGLAB (www.sccn.ucsd.edu/eeglab). Filtering was set with a low-pass at $20 \mathrm{~Hz}$ and the data were re-referenced to the average mastoid. The data were re-sampled to $250 \mathrm{~Hz}$. Independent components analysis was used to extract out eye-blink and eye movements within the data. Individual ERP segmentation began $100 \mathrm{~ms}$ before, and continued $900 \mathrm{~ms}$ post-stimulus onset. All segments were baseline corrected and averaged. ERP segments were time locked to the onset of the flanker.

\section{STATISTICAL ANALYSIS}

\section{Behavioral performance}

Behavioral analyses examined the effect of RT and accuracy using repeated measure ANOVAs. Only correct responses to the flanker trials were used in the analysis. The flanker data were analyzed using a $2 \times 3$ factor design: congruence (congruent or incongruent) $\times$ load (single flanker, set size 4 , or set size 7 ). The Sternberg data were analyzed using a $2 \times 2$ factor design: load (single or dual) $\times$ set size (four or seven items). Follow-up paired $t$-test comparisons investigated significant interactions. Only correct responses were used in the RT analysis.

\section{Event-related potentials}

Electrophysiological analyses examined the P1 and P300 in the stimulus-locked waveform. Only trials with correct responses were used in the analysis. The P1 was identified as the first positive peak in the time window of 110-130 ms at electrodes O1 and O2. Mean amplitudes in the 110- to 130-ms interval were measured across both occipital electrodes. The P300 was identified as the large positive component that occurred between 350 and $600 \mathrm{~ms}$. The ERP mean amplitude measures for P1 and P300 were then submitted separately to two-way ANOVAs that examined congruence (congruent or incongruent) $\times$ load (single flanker, set size 4 , or set size 7 ).

\section{RESULTS}

\section{BEHAVIORAL}

\section{Flanker task}

For RTs, a main effect of congruence was found, indicating that participants were faster to respond to congruent flankers compared to incongruent flankers $[F(1,14)=58.053, p<0.001$; see Figure 2A $]$. Only a marginal effect was found for load $[F(2,28)=2.679$, $p=0.086]$. The trend is consistent with the observation that the fastest RTs occurred during the single flanker condition ( $411.5 \mathrm{~ms}$ ) followed by the dual task condition with Sternberg set sizes of 4 (423 $\mathrm{ms}$ ) and 7 (430 ms). The interaction between congruence and load was not significant $(p=0.95)$, indicating that the addition of a working memory task did not alter the flanker interference effect.

Overall accuracy in the flanker task was fairly high ( mean $=91 \%$ ). The main effect of congruence reflected the fact that accuracy was higher when flankers were congruent, relative to incongruent

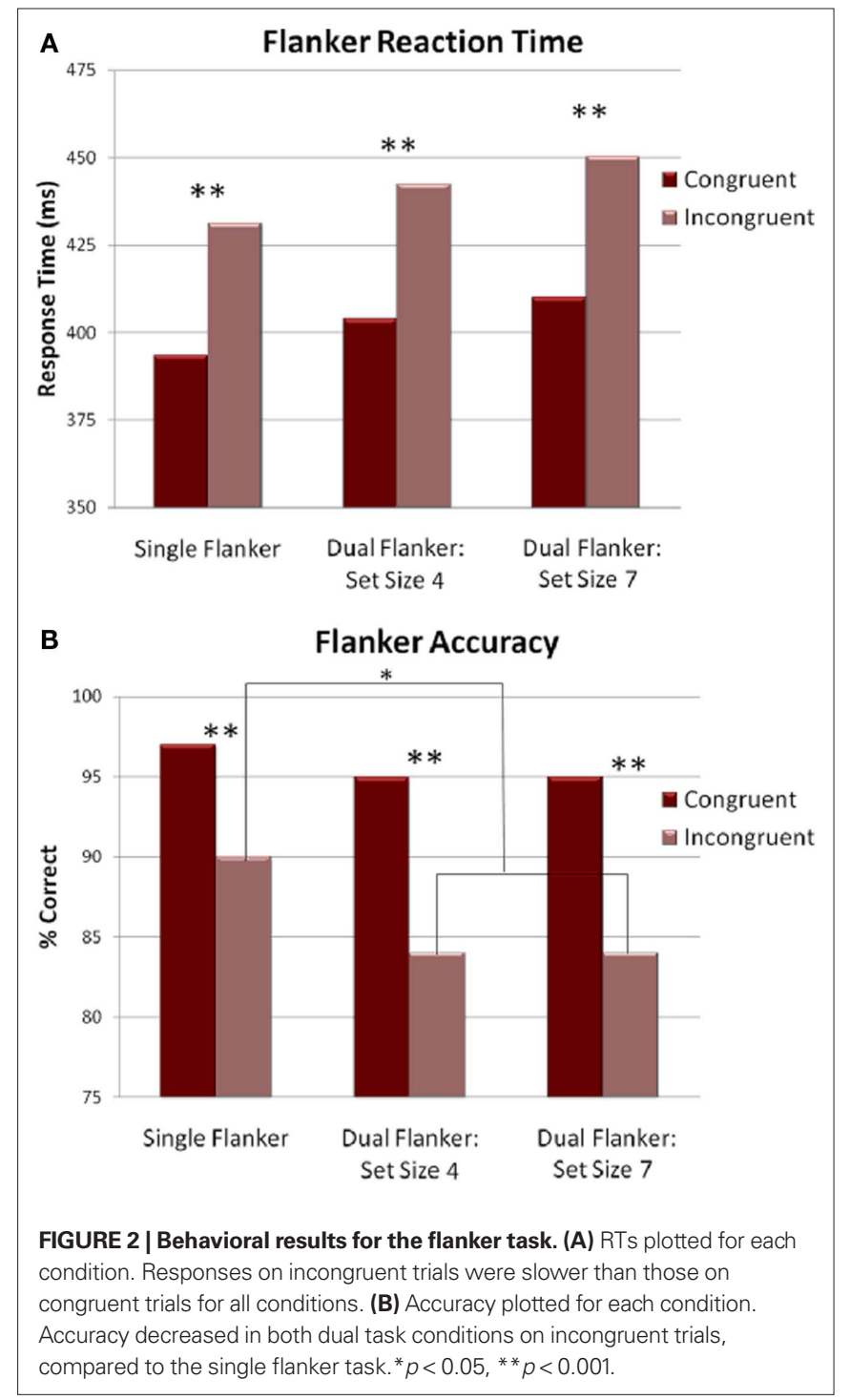

$[F(1,14)=56.934, p<0.001]$. In addition, a main effect of load suggests that responses were more accurate in the single task condition than when combined with a Sternberg set of four or seven $[F(2,28)=5.780, p=0.026]$. The main effects were followed by a significant interaction of congruence $\times$ load $[F(2,28)=13.293$, $p<0.001]$. Follow-up comparisons revealed that the flanker interference effect was greater in the dual task conditions. Responses were more accurate on incongruent trials in the single flanker condition compared to the dual task with set size $4[t(14)=3.301, p=0.005]$ or set size $7[t(14)=3.280, p=0.005$; see Figure $2 B]$. There was no significant effect of load when the flankers were congruent [single flanker versus set size 4 : $t(14)=0.891$, ns; single flanker versus set size 7: $t(14)=1.271$, ns; set size 4 versus set size $7: t(14)=1.096, \mathrm{~ns}]$.

Additional correlation analyses were conducted to test whether a speed versus accuracy trade-off occurred. We expected that load might have affected the error rate, which in turn was inversely related to the speed of the response. The stimuli appeared at a very rapid rate and to adjust for a higher error rate, participants might have slowed down. To test this, we used Pearson correlations for 
RT and accuracy for each of the load conditions. Results indicated that participants did make speed/accuracy trade-offs, but only in the most difficult conditions. A significant correlation was found for incongruent trials for set size $4(r=0.519, p=0.047)$ and set size 7 ( $r=0.801, p<0.001)$, but not for single flanker. When the task became more difficult (i.e., incongruent flankers), longer RTs were associated with an increase in accuracy.

\section{Sternberg task}

For RTs, a main effect of set size indicated that responses to the memory probes were significantly faster when there were only four items in the set as opposed to seven $[F(1,13)=28.461, p<0.001]$. There was neither a main effect of task $(p=0.50)$ nor an interaction $(p=0.44)$, which suggests that the addition of an attention task did not alter RTs to memory probes (see Figure 3A).

The analysis of accuracy on Sternberg trials revealed a significant main effect of load $[F(1,13)=12.429, p=0.003]$ indicating that responses were more accurate when completing the Sternberg trials

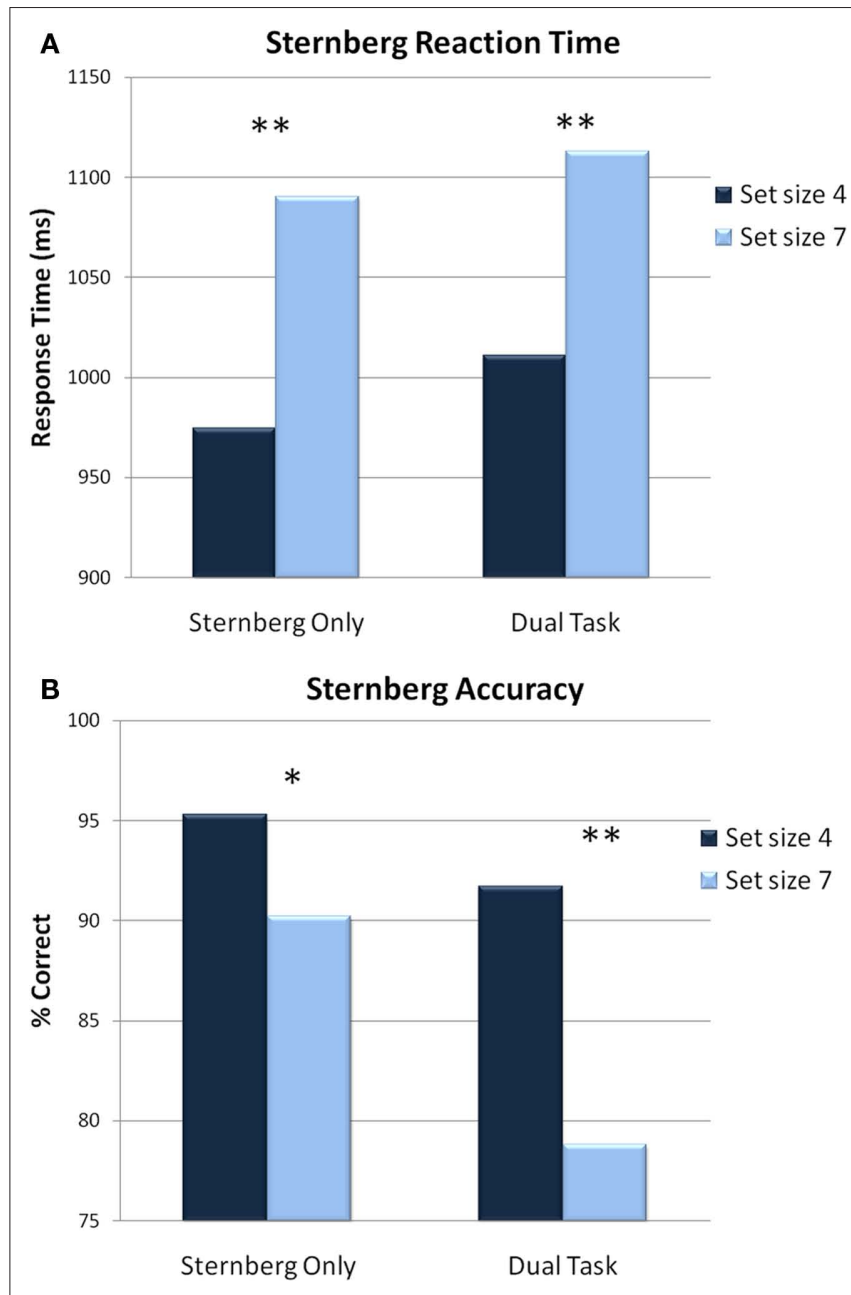

FIGURE 3 | Behavioral results for the Sternberg task. (A) RTs on Sternberg trials for each condition. Participants responded faster to probes from a set size of 4 compared to set size of 7. (B) Accuracy on Sternberg trials for each condition. Accuracy declined to probe items from a set size of 7 compared to a set size of $4 .{ }^{*} p<0.05,{ }^{*} p<0.001$. alone, compared to when the flanker trials intervened. The analysis also revealed a significant main effect of set size indicating that participants were more accurate when there were only four items in memory set compared to seven $[F(1,13)=27.533, p<0.001]$. This was followed by a significant interaction of load $\times$ set size $[F(1,13)=24.548, p<0.001$; see Figure 3B $]$. Follow-up comparisons indicated that accuracy declined in the dual task condition only when the set size contained seven items $[t(13)=-5.115$, $p<0.001]$. Participants were significantly more accurate when responding to probes from set size 4 compared to set size 7 in both the single $[t(13)=-2.789, p=0.015]$ and dual task conditions $[t(13)=-6.585, p<0.001]$.

\section{EVENT-RELATED POTENTIALS \\ P1}

The $\mathrm{P} 1$ was quantified across electrodes $\mathrm{O} 1$ and $\mathrm{O} 2$ as the most positive peak occurring between 110 and $130 \mathrm{~ms}$ after stimulus onset. The mean amplitude during this time window was averaged across both electrodes and analyzed in a two-way ANOVA. A significant main effect of load $[F(2,28)=7.423, p=0.007]$ indicated that $\mathrm{P} 1$ amplitude decreased when the Sternberg task was included, relative to the single task condition (see Figure 4). P1 amplitude was significantly larger in the single flanker compared to the dual task conditions with either four $[t(14)=2.893, p=0.012]$ or seven items $[t(14)=3.21, p=0.006]$, whereas the latter two conditions did not differ from each other $[t(14)=-0.908, \mathrm{~ns}]$. There was neither a main effect of congruence $[F(1,14)=0.008, \mathrm{~ns}]$ nor a significant interaction of load $\times$ congruence $[F(2,28)=0.536, \mathrm{~ns}]$.

\section{P300}

Mean amplitudes between 300 and 650 ms were examined with an initial three-way ANOVA including the midline electrodes (electrode: $\mathrm{Fz}, \mathrm{FCz}, \mathrm{Cz}, \mathrm{CPz}, \mathrm{Pz}, \mathrm{POz}$ ), congruence (congruent, incongruent), and load (single flanker, set size 4 , set size 7 ) as factors. A significant main effect of electrode $[F(5,70)=8.031, p<0.007]$ was found. The largest $\mathrm{P} 300$ amplitude was observed at $\mathrm{CPz}$, which was the focus of subsequent analyses. A two-way ANOVA at $\mathrm{CPz}$ showed a main effect of congruence $[F(1,14)=4.633, p=0.049]$ that indicated larger positive amplitudes to incongruent versus congruent trials. A marginally significant effect of load $[F(2,28)=3.681$, $p=0.073]$ suggested that the single flanker condition elicited the largest amplitude response $(7.01 \mu \mathrm{V})$, followed by the dual task conditions with set size $4(5.984 \mu \mathrm{V})$ and set size $7(5.4 \mu \mathrm{V})$. In addition, a significant interaction between load and congruence $[F(2,28)=3.709, p=0.04]$ was observed. Follow-up comparisons revealed that incongruent flankers produced a larger P300 in the single task condition, compared to the dual task conditions with set size $4[t(14)=2.178, p=0.047]$ or set size $7[t(14)=2.798$, $p=0.014$; see Figure 5].

\section{DISCUSSION}

The current study tested the effects of working memory load on attentional control and conflict resolution using a dual task design. The results demonstrated that the concurrent task demands of maintaining items in working memory diminished the ability to attend to targets and ignore distractors in a flanker interference task. Incongruent flanker stimuli were more difficult to ignore when 

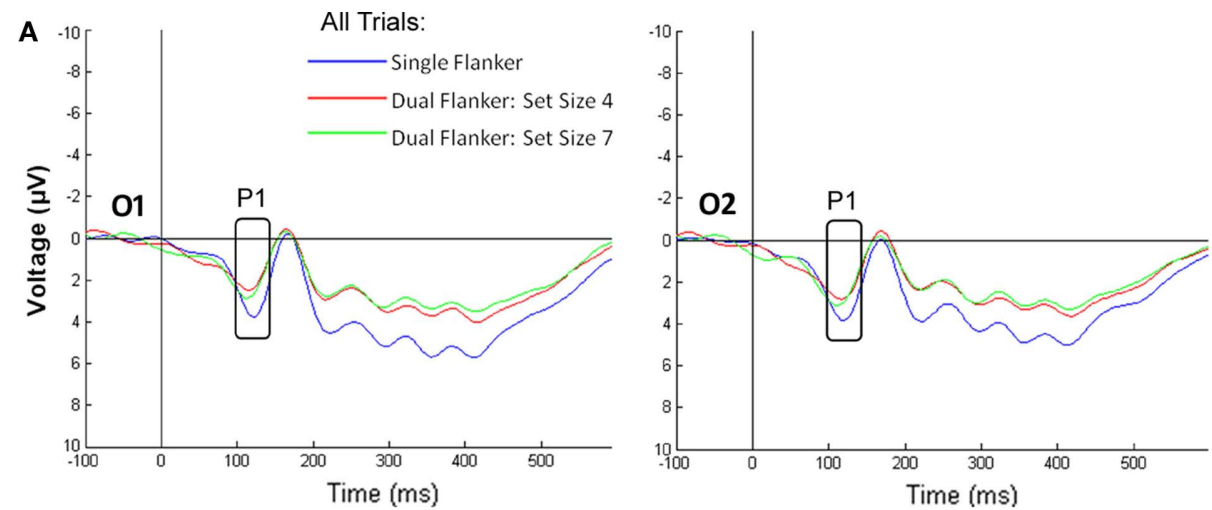

B


FIGURE 4 | P1 in response to load. (A) The dual task conditions significantly diminished P1 amplitude at electrode O1 and O2, compared to the P1 amplitude for the single task. (B) Topographic maps display the scalp voltage distribution at $120 \mathrm{~ms}$, indicating that the P1 is located over both $\mathrm{O} 1$ and $\mathrm{O} 2 \mathrm{electrodes}$ for each task condition.

working memory load increased, as indicated by reduced behavioral accuracy and decreased P300 amplitude. This suggests that working memory is needed to filter out irrelevant information, selectively focus attention and resolve response conflict. Furthermore, the convergence of behavioral and ERP results supports the integral role of working memory in directing and regulating attentional selection, in support of the cognitive load theory (Lavie et al., 2004).

As observed in many prior studies, accuracy was lower when the flankers were incongruent relative to congruent (Eriksen and Eriksen, 1974; Kopp et al., 1996). This performance deficit was worsened when participants were required to maintain either four or seven items in working memory during the flanker task. Previous research has found slower RTs in flanker-type tasks when working memory load was high and distractors were incongruent (Lavie, 2005; Lavie and De Fockert, 2005). Although our current RT results did not show greater flanker interference with added working memory demands, the accuracy results indicated that incongruent distractors did cause greater interference when working memory was loaded. The lack of an RT interference effect may be due in part to the short trial and inter-trial durations. The flanker task was fast-paced, which led to a significant speed/accuracy trade-off in the dual task conditions.

Compared to working memory performance in the single task condition, accuracy in the dual task Sternberg decreased, but only for memory sets with seven items. This suggests that the dual task design produced deficits in working memory when attentional demands were instituted during the delay period, but only for the high load condition. The current results also suggest that divided attention is detrimental to working memory, especially under more difficult conditions (Kane and Engle, 2003). This is consistent with Gazzaley's (2011) recent review of the literature showing that as working memory load increases, attentional capacity decreases, and in turn, causes working memory performance to decline.

Event-related potential recordings provided evidence for electrophysiological changes associated with dual task processing. Both P1 and P300 showed significant decreases in amplitude in the flanker task when working memory demands were increased. Early changes in the P1 indicated that regardless of distractor congruity, initial visual processing was diminished when working memory was taxed. This decline in amplitude occurred regardless of the number of items held in working memory. The later P300 component also decreased significantly when working memory was loaded, but only for trials with incongruent flanker stimuli.

Previous research suggests that $\mathrm{P} 1$ generally increases in amplitude to targets appearing in an attended location and decreases to targets at an unattended location (Hillyard et al., 1998; Hopfinger et al., 2000; Luck et al., 2000). In the current study, attention was divided between the flanker stimuli and the contents of working memory during the dual task conditions. Coordination of dual task performance utilized resources typically involved in modulating early visual processing. Importantly, the early onset of the P1 effect suggests that top-down processes in PFC influence visual attention within 110-130 ms of stimulus presentation. Neuropsychological studies have provided direct evidence for this modulatory effect. Patients with lesions of PFC show reductions in early visual components, such as the N1 and N2 (Knight, 1997), the left lateralized N170 to words (Swick, 1998), and the P1 (Barceló et al., 2000; Yago et al., 2004). These lesion results suggest that PFC normally provides 


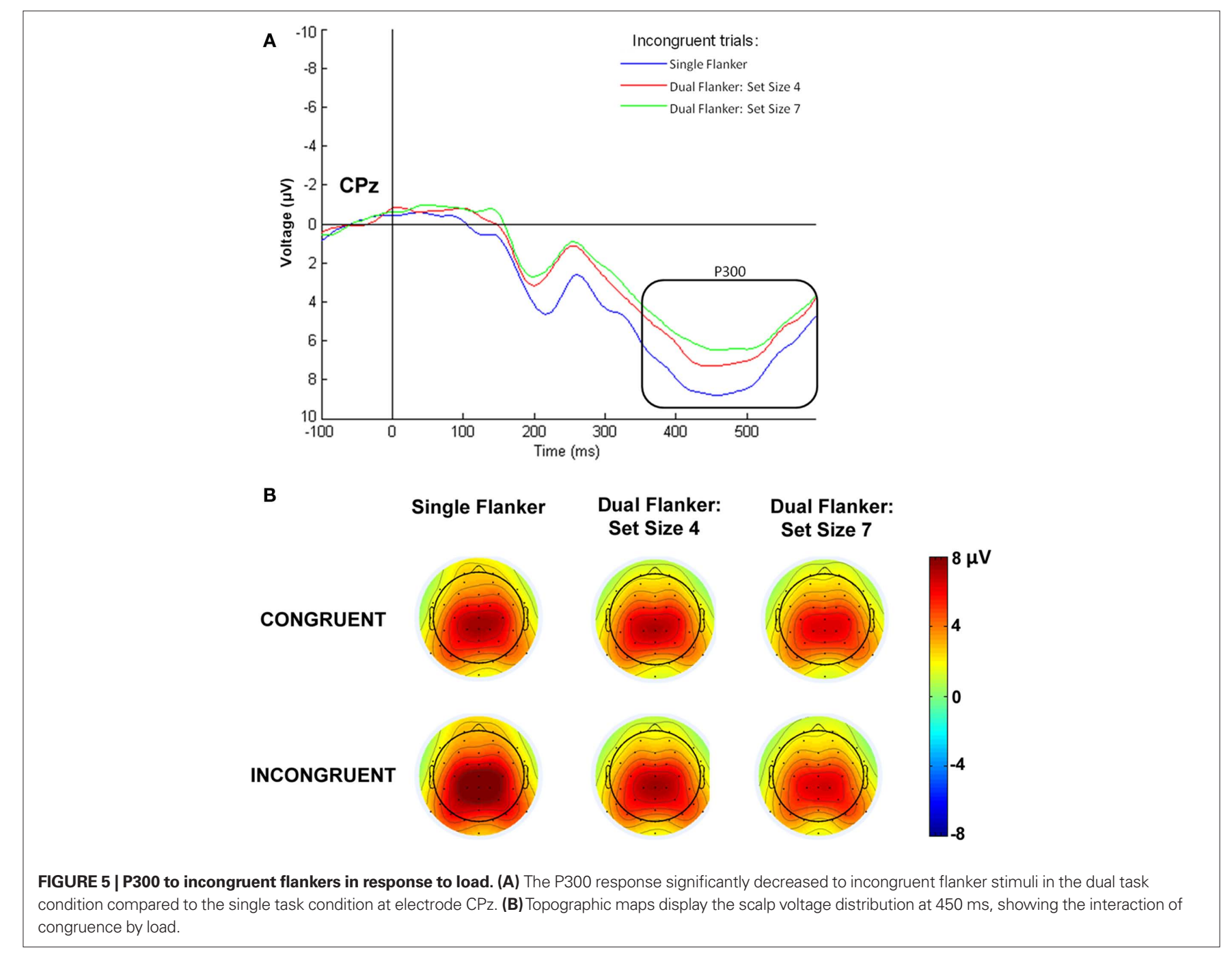

an excitatory input to boost attentionally relevant processing in extrastriate cortex. Therefore, when working memory and dual task demands tax the PFC in healthy controls, it is likely that fewer resources are available to modulate the $\mathrm{P} 1$ response in extrastriate cortex. As other researchers have found that P1 decreases as a function of attention (Hillyard et al., 1998; Luck et al., 2000), our current findings suggest that attention decreases when items are maintained in working memory, and that this may be the result of less top-down activation from the PFC.

Previous fMRI results demonstrated an increase in activation in extrastriate regions when working memory load was high compared to low (de Fockert et al., 2001), a finding that has recently been extended to primary visual cortex (Kelley and Lavie, 2010). de Fockert et al. (2001) suggested that increased activity in visual cortex reflected difficulty in ignoring irrelevant stimuli. That is, visual regions were more active when working memory load was high and distractors were present (de Fockert et al., 2001). In the current study, P1 was not sensitive to distractor processing (i.e., no effect of flanker congruence). The difference between our results and those of de Fockert et al. (2001) may relate to the temporal resolution of the methods used. As mentioned before, fMRI provides good spatial resolution of brain activation to specific stimuli, but is unable to provide good temporal resolution. Thus, it is unknown whether the extrastriate activation reflects early or late changes in visual processing. In contrast, our findings suggest that within the first $100 \mathrm{~ms}$, the response of visual cortex to attentionally relevant stimuli is diminished when items are maintained in working memory.

In contrast to the $\mathrm{P} 1$, the $\mathrm{P} 300$ component showed a decrement in the dual task conditions only when irrelevant stimuli were present (similar to performance accuracy). Previous reports have indicated that P300 amplitude decreases as a function of task difficulty (Picton, 1992; Garcia-Larrea and Cezanne-Bert, 1998; Wintink et al., 2001). In dual task conditions, P300 reflects the amount of resources available for the current task (Sirevaag et al., 1989; Singhal and Fowler, 2004), and P300 amplitude decreases in the secondary task as the difficulty of the primary task increases. As predicted, we observed a reduction in P300 amplitude on incongruent trials when working memory load increased. This is consistent with the notion that increased complexity of task demands caused greater distribution of attentional resources and therefore, a reduction in recruitment of the generators of the P300 (Wintink et al., 2001). 
The interaction of load and congruity indicated that demands on working memory influenced the P300 amplitude to incongruent flankers only. As suggested by Lavie and colleagues (de Fockert and Lavie, 2001; Lavie et al., 2004; Lavie, 2005; Lavie and de Fockert, 2005), working memory functions to selectively focus attention on the target and reduce the intrusion of irrelevant distractors. The current P300 findings support this hypothesis and suggest that interference from incongruent flankers was more difficult to process when working memory capacity was full (de Fockert et al., 2001). Controlling attention during multiple tasks requires the frontal executive component to coordinate planning and attention to goal-relevant stimuli (Garcia-Larrea and Cezanne-Bert, 1998). Although lesions studies have suggested that the P300 recorded in simple target detection tasks does not have neural sources in PFC (Knight, 1997), P300 amplitude reductions have been observed in PFC patients during more difficult categorization tasks (Swick, 1998). Therefore, the decrease in P300 amplitude in the dual task conditions could reflect a decline in frontal-dependent measures of attentional control (Garcia-Larrea and Cezanne-Bert, 1998).

One limitation of the results was the inclusion of flanker arrows above, below, and both above and below the target arrow. The number of flankers in the display may have contributed to a perceptual load effect. According to Lavie et al. (2004) increasing perceptual load (number of items in the display) reduces the amount of interference caused by distractors. In contrast, increasing cognitive load causes greater interference in processing distractors (Lavie et al., 2004). The purpose of the current study was to examine the effects of cognitive load on attentional processing and ERP components. Flankers located both above and below the central target arrow could be considered a greater perceptual load than flankers either below or above the target arrow. In order to reduce the effect of perceptual load and only examine cognitive load, we collapsed across

\section{REFERENCES}

Awh, E., Anllo-Vento, L., and Hillyard, S. (2000). The role of spatial selective attention in working memory for locations: evidence from eventrelated Potentials. J. Cogn. Neurosci. 12, 840-847.

Barceló, F., Suwazono, S., and Knight, R. T. (2000). Prefrontal modulation of visual processing in humans. Nat. Neurosci. 3, 399-403.

Bledowski, C., Prvulovic, D., Goebel, R., Zanella, F. E., and Linden, D. E. (2004). Attentional systems in target and distractor processing: a combined ERP and fMRI study. Neuroimage 22, 530-540.

Corbetta, M., and Shulman, G. L. (2002). Control of goal-driven and stimulus driven attention in the brain. Nat. Rev. Neurosci. 3, 201-215.

de Fockert, J. W., Rees, G., Frith, C. D., and Lavie, N. (2001). The role of working memory in visual selective attention. Science 291, 1803-1806.

Di Russo, F., Martinez, A., Sereno, M. I., Pitzalis, S., and Hillyard, S. A. (2001). Cortical sources of the early components of the visual evoked potential. Hum. Brain Mapp. 15, 95-111.

Downing, P. E. (2000). Interactions between visual working memory and selective attention. Psychol. Sci. 11, 467-473.

Duncan, C. C., Barry, R. J., Connolly, J. F., Fischer, C., Michie, P. T., Naatanen, R., Polich, J., Reinvang, I., and Van Petten, C. (2009). Event-related potentials in clinical research: guidelines for eliciting, recording, and quantifying mismatch negativity, P300, and N400. Clin. Neurophysiol. 120, 1883-1908.

Eriksen, B. A., and Eriksen, C. W. (1974). Effects of noise letters upon the identification of a target letter in a nonsearch task. Percept. Psychophys. 16, 143-149.

Garcia-Larrea, L., and Cezanne-Bert, G. (1998). P3, positive slow wave and working memory load: a study on the functional correlates of slow wave activity. Electroencephalogr. Clin. Neurophysiol. 108, 260-273.

Gazzaley, A. (2011). Influence of early attentional modulation on working memory. Neuropsychologia 49, 1410-1424. arrow location to selectively examine cognitive load only. Future studies should examine the electrophysiological changes associated with perceptual load processing and the interaction of perceptual load and cognitive load on attention.

\section{CONCLUSION}

The present study illustrates the detrimental effects of dual task processing and cognitive control. High working memory load interfered with the attentional control network, especially when attention was needed to filter out irrelevant distractors. Our findings extend the work of Lavie and colleagues (de Fockert and Lavie, 2001; Lavie et al., 2004; Lavie, 2005; Lavie and de Fockert, 2005) by revealing the time-course of load effects on the brain regions supporting visual attention and conflict resolution. The early extrastriate $\mathrm{P} 1$ response was sensitive to increases in cognitive load regardless of distractor congruity. We suggest that working memory demands decreased top-down modulatory influences from PFC as early as $100 \mathrm{~ms}$. The later P300 response was sensitive to both increased cognitive load and the presence of distracting flanker stimuli. We suggest that the decrease in P300 amplitude reflects the diminished availability of resources to selectively focus attention and resolve response conflict. The present findings support and extend the evidence for the necessity of working memory in resolving response conflicts and attention. Future studies in patients with PFC lesions, or lesions in white matter tracts connecting frontal and posterior association cortices, will be helpful in understanding the importance of frontal projections on top-down attention processing and cognitive control.

\section{ACKNOWLEDGMENTS}

This work was supported by a VA Merit Review grant, the U.S. Army Medical Research and Materiel Command under W81XWH-08-2-0086, and NSF grant 023681.
Gazzaley, A., Clapp, W., Kelley, J., McEvoy, K., Knight, R. T., and D'Esposito, M. (2008). Age-related top-down suppression deficit in the early stages of cortical visual memory processing. Proc. Natl. Acad. Sci. U.S.A. 105, 13122-13126.

Handy, T. C., Soltani, M., and Mangun, G. R. (2001). Perceptual load and visuocortical processing: event-related potentials reveal sensory-level selection. Psychol. Sci. 12, 213-218.

Hillyard, S. A., and Anllo-Vento, L. (1998). Event-related brain potentials in the study of visual selective attention. Proc. Natl. Acad. Sci. U.S.A. 95, 781-787.

Hillyard, S. A., Vogel, E. K., and Luck, S. J. (1998). Sensory gain control (amplification) as a mechanism of selective attention: electrophysiological and neuroimaging evidence. Philos. Trans. R. Soc. Lond. B Biol. Sci. 353, 1257-1270.

Hopf, J. M., and Mangun, G. R. (2000). Shifting visual attention in space: an electrophysiological analysis using high spatial resolution mapping. Clin. Neurophysiol. 111, 1241-1257.
Hopfinger, J. B., Buonocore, M. H., and Mangun, G. R. (2000). The neural mechanisms of top-down attentional control. Nat. Neurosci. 3, 284-291.

Jasper, H. H. (1958). The ten-twenty electrode system of the International Federation. Electroencephalogr. Clin. Neurophysiol. 10, 371-375.

Kane, M. J., and Engle, R. W. (2003). Working-memory capacity and the control of attention: the contributions of goal neglect, response competition and task set to stroop interference. $J$. Exp. Psychol. Gen. 132, 47-70.

Kelley, T.A., and Lavie, N. (2010). Working memory load modulates distractor competition in primary visual cortex. Cereb. Cortex 21, 659-665.

Knight, R. T. (1997). Distributed cortical network for visual attention. J. Cogn. Neurosci. 9, 75-91.

Kopp, B., Rist, F., and Mattler, U. (1996). N200 in the flanker task as a neurobehavioral tool for investigating executive control. Psychophysiology 33, 282-294.

Krawczyk,D.C.,Gazzaley,A., andD'Esposito, M. (2007). Reward modulation of pre- 
frontal and visual association cortex during an incentive working memory task. Brain Res. 1141, 168-177.

Lavie, N. (2005). Distracted and confused? Selective attention under load. Trends Cogn. Sci. (Regul. Ed.) 9, 75-82.

Lavie, N., and De Fockert, J. (2005). The role of working memory in attentional capture. Psychon. Bull. Rev. 12, 669-674.

Lavie, N., Hirst, A., de Fockert, J. W., and Viding, E. (2004). Load theory of selective attention and cognitive control. J. Exp. Psychol. Gen. 133, 339-354.

Luck, S. J., Woodman, G. F., and Vogel, E. K. (2000). Event-related potential studies of attention. Trends Cogn. Sci. (Regul. Ed.) 4, 432-440.

Picton, T.W. (1992). The P300 wave of the human event-related potential. J. Clin. Neurophysiol. 9, 456-479.

Polich, J., and Kok, A. (1995). Cognitive and biological determinants of P300: an integrative review. Biol. Psychol.41, 103-146.

Singhal, A., and Fowler, B. (2004). The differential effects of Sternberg shortand long-term memory scanning on the late Nd and P300 in a dual-task paradigm. Brain Res. Cogn. Brain Res. 21, 124-132.

Sirevaag, E. J., Kramer, A. F., Coles, M. G., and Donchin, E. (1989). Resource reciprocity: an event-related brain potentials analysis. Acta Psychol. (Amst.) 70, 77-97.

Soto, D., Heinke, D., Humphreys, G. W., and Blanco, M. J. (2005). Early, involuntary top-down guidance of attention from working memory. J. Exp. Psychol. Hum. Percept. Perform. 31, 248-261.

Swick, D. (1998). Effects of prefrontal lesions on lexical processing and repetition priming: an ERP study. Brain Res. Cogn. Brain Res. 7, 143-157.
Watter, S., Geffen, G. M., and Geffen, L. B. (2001). The n-back as a dual-task: P300 morphology under divided attention. Psychophysiology 38, 998-1003.

Wintink, A. J., Segalowitz, S. J., and Cudmore, L. J. (2001). Task complexity and habituation effects on frontal P300 topography. Brain Cogn. 46, 307-311.

Yago, E., Duarte, A., Wong, T., Barceló, F., and Knight, R. T. (2004). Temporal kinetics of prefrontal modulation of the extrastriate cortex during visual attention. Cogn. Affect. Behav. Neurosci. 4, 609-617.

Yantis, S. (2000). "Goal-directed and stimulus-driven determinants of attentional control," in Attention and Performance XVIII: Control of Cognitive Operations, eds S. Monsell and J. Driver (Cambridge, MA: MIT Press), 73-104.
Conflict of Interest Statement: The authors declare that the research was conducted in the absence of any commercial or financial relationships that could be construed as a potential conflict of interest.

Received: 18 February 2011; paper pending published: 10 March 2011; accepted: 25 May 2011; published online: 13 June 2011. Citation: Pratt N, Willoughby A and Swick $D$ (2011) Effects of working memory load on visual selective attention: behavioral and electrophysiological evidence. Front. Hum. Neurosci. 5:57. doi: 10.3389/ fnhum.2011.00057

Copyright () 2011 Pratt, Willoughby and Swick. This is an open-access article subject to a non-exclusive license between the authors and Frontiers Media SA, which permits use, distribution and reproduction in other forums, provided the original authors and source are credited and other Frontiers conditions are complied with. 